\title{
Chronic relapsing idiopathic polyneuropathy with primary axonal lesions
}

\author{
J JULIEN,* C VITAL, $\dagger$ A LAGUENY,* X FERRER,* C BRECHENMACHER $\dagger$ \\ From the Department of Neurology, ${ }^{*}$ Hopital du Haut-Lévêque, and the Department of Neuropathology, $\dagger$ CHU \\ Bordeaux, France
}

SUMMARY Idiopathic polyradiculoneuropathy with primary axonal lesions is rarely encountered. Two cases are reported with a chronic relapsing course and a fatal outcome. Neuropathological examination of biopsied peripheral nerve in the two patients and in a necropsy case showed loss of myelinated fibres, but neither active demyelination nor inflammatory cells were observed. Acute and chronic relapsing axonal polyradiculoneuropathies appear to be two clinical forms of a peculiar entity different from GBS

Guillain Barré syndrome (GBS) is typically characterised by an acute demyelinating polyradiculoneuropathy which progresses to maximum weakness within 2 to 3 weeks of onset and recovers in most cases after 6 to 12 months.'

Another type of idiopathic demyelinating polyradiculoneuropathy may be observed with similar clinical, electrophysiological and pathological features, but with a protracted initial phase and a chronic monophasic or relapsing course. ${ }^{2}$

Idiopathic polyradiculoneuropathy with primary axonal lesions is rarely encountered. Its course may be acute $^{3}$ or chronic relapsing. ${ }^{4}$ Electrophysiological and pathological signs are quite different from the demyelinating forms and prognosis is generally poor.

We report two cases with chronic relapsing polyradiculoneuropathy, where peripheral nerve biopsy and in one case necropsy disclosed primary axonal lesions.

\section{Case reports}

Case 1 A 54 year old woman had been suffering from progressive motor paresis for 6 months when she was examined for the first time in January 1985 . She presented with a moderate proximal and distal weakness of all limbs with wasting of the hand muscles. All reflexes were absent. Hypaesthesia was noted for pin prick and touch with sock and glove distribution. Cranial nerves were normal but

Address for reprint requests: Dr J Julien, Department of Neurology, Hôpital du Haut-Lévêque, CHU Bordeaux, 33604 Pessac, France.

Received 29 July 1988 and in revised form 9 January 1989. Accepted 24 February 1989 breathing was weak and she was dyspnoeic when walking. CSF protein was $90 \mathrm{mg} / 100 \mathrm{ml}$ with a normal level of $\gamma$ globulin. Routine analysis was normal as was serum electrophoresis. Porphyrin screening, cryoglobulins, anti-DNA and anti-HbS antibodies were negative. Motor nerve conduction velocity measured in the common peroneal nerve was 45.2 $\mathrm{m} / \mathrm{s}$ with a $6.4 \mathrm{~ms}$ distal latency. Proximal motor nerve conduction velocity ( $\mathrm{F}$-wave conduction) stimulating the ulnar nerve at the wrist was slowed $(30 \cdot 3 \mathrm{~m} / \mathrm{s})$ while the Fwave was normal when stimulating the tibial nerve at the ankle $(30 \cdot 3 \mathrm{~m} / \mathrm{s})$. Chronic partial denervation was found in all limbs. Sensory action potential was reduced in amplitude in the ulnar nerve $(6 \mu \mathrm{v})$. Three months later, another electrophysiological study disclosed fibrillation potentials at rest in all the examined muscles. Motor nerve conduction velocity measured on the left peroneal nerve was $40 \mathrm{~m} / \mathrm{s}$ with a $5.3 \mathrm{~ms}$ distal latency.

Superficial peroneal nerve biopsy disclosed no abnormality in the interstitium. Direct immunofluorescence was negative for IgA, IgG, IgM, fibrinogen and C3. Some sparse ovoids were observed on semi-thin sections. A histogram showed $7000 / \mathrm{mm}^{2}$ myelinated fibres with a marked reduction of the largest myelinated fibres. On ultrastructural study, there were some bands of Büngner but no features of active demyelination or any inflammatory cells (figs 1 and 2).

The patient was treated with prednisone $(50 \mathrm{mg} /$ daily $)$ without improvement. A few weeks later, three 31 plasma exchanges were given over one week, then three 31 plasma exchanges over the next 3 weeks. A moderate improvement was observed. The patient was no longer dyspnoeic and was able to walk without assistance. Azathioprine ( $200 \mathrm{mg} /$ daily) was administered and she was discharged in March 1985

She relapsed in November 1985, was again unable to walk, and she presented with respiratory failure. Her condition continued to worsen progressively and in January 1986, she developed difficulty in swallowing and breathing; she was now virtually quadriplegic. She was intubated and placed on a respirator. Eight 31 plasma exchanges were given in 3 


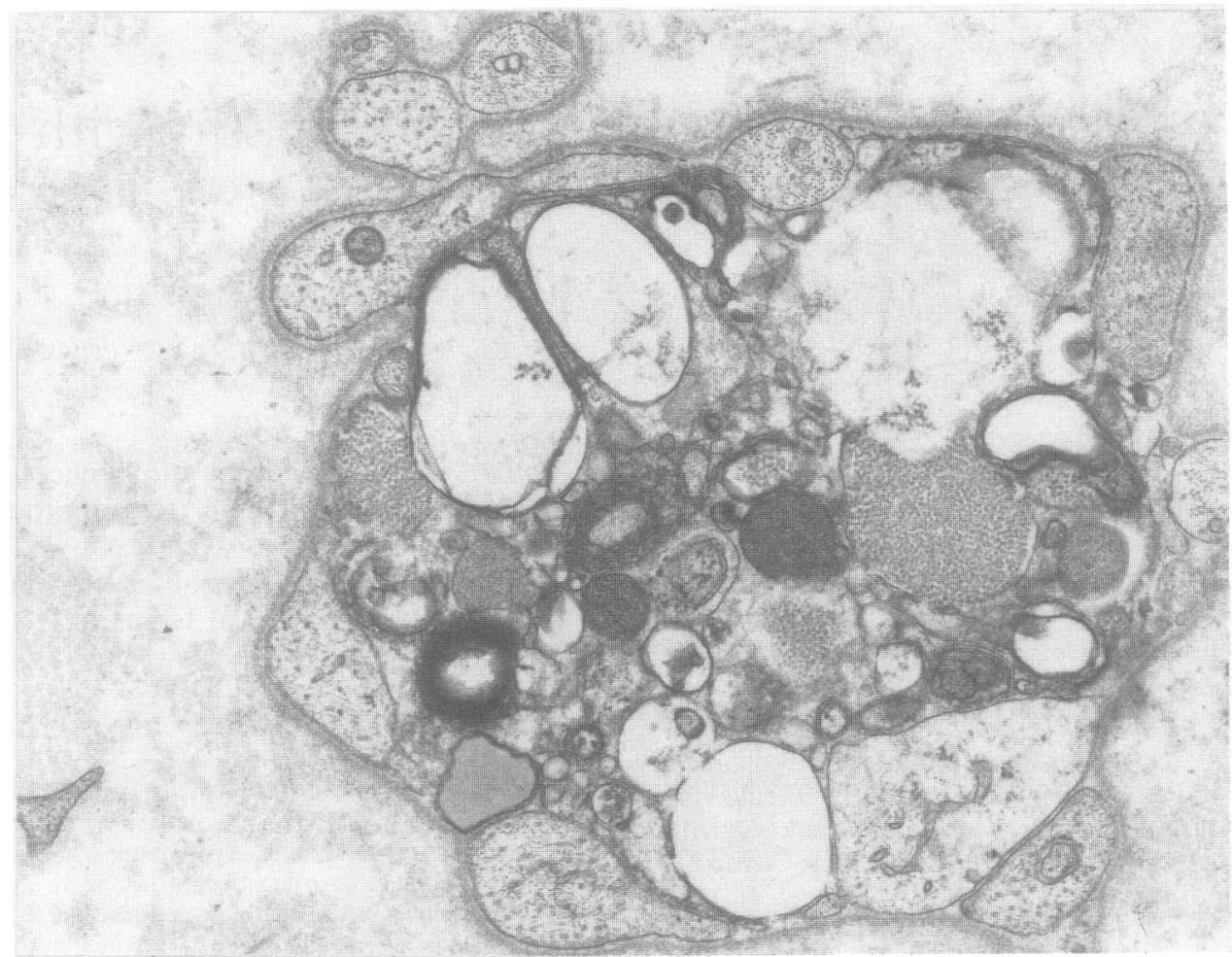

Fig 1 Electron micrograph shows a band of Büngner with several axonal sprouts at the periphery $(\times$ 21,000).

weeks. A slow improvement appeared and the patient was weaned off the respirator in June 1986. The following September, she was again able to walk with sticks. However, in November, a third relapse occurred within four days, leaving her bedridden, quadriplegic and requiring ventilatory assistance. From this time, the patient remained in a serious condition and died one year later in November 1987.

Necropsy disclosed a left ventricular hypertrophy without ischaemic lesions and pulmonary congestion with suppurated alveolitis. There was no malignancy. On transverse spinal cord sections at the cervical, thoracic and lumbar levels, the nerve roots did not show any inflammatory cells. A slight cellular loss was observed in the dorsal root ganglia while some other neurons were atrophic. Anterior horn cells did not exhibit central chromatolysis. The nerve roots did not show any demyelinating lesions under light microscopic study.

Case 2 A 62 year old man complained of weakness in his right arm on 6 September 1983. The deficit spread in six weeks to all limbs. When the patient was admitted there was marked proximal weakness of all limbs, and he was unable to stand. Reflexes were absent. There was no hypaesthesia nor muscle wasting. In the CSF, proteins were $81 \mathrm{mg} / 100 \mathrm{ml}$. Routine studies and serum electrophoresis were normal. Porphyrin and cryoglobulin screening were negative. The histocompatibility antigen was B27. Electrophysiological studies showed fibrillation potentials at rest in all the examined muscles. Motor nerve conduction velocity was slow $(25 \mathrm{~m} / \mathrm{s}$ in the left median nerve) with a $8 \mathrm{~ms}$ distal latency. The left common peroneal nerve was inexcitable.

A peripheral nerve biopsy was carried out on the right superficial peroneal nerve in January 1984 and on the left in August 1984. On the histogram performed on semi-thin sections, the density of myelinated fibres was reduced to 8600 fibres $/ \mathrm{mm}^{2}$ in the first peripheral nerve biopsy, and to 7600 fibres $/ \mathrm{mm}^{2}$ in the second. Direct immunofluorescence was negative for IgA, IgG, IgM, fibrinogen and C3. Teased fibres study was performed on the first biopsy. Twenty seven isolated fibres were studied: there were two fibres with internodal demyelination without any ovoids or balls in the cytoplasm of the associated schwann cells. On ultrastructural studies, there were a few isolated remyelinating fibres with a myelin which was too thin compared to axonal size. One of these remyelinating fibres was surrounded by concentric debris of schwann cell basal lamina. There were rare myelin and axonal lesions with the presence of bands of Büngner; several clusters of regeneration of myelinated fibres were observed. Axonal lesions were rarely observed with an enlarged axon stripped of myelin (fig 3). The presence of small axonal sprouts in the same schwann cell cytoplasm indicated a regenerating process after axonal degeneration. 


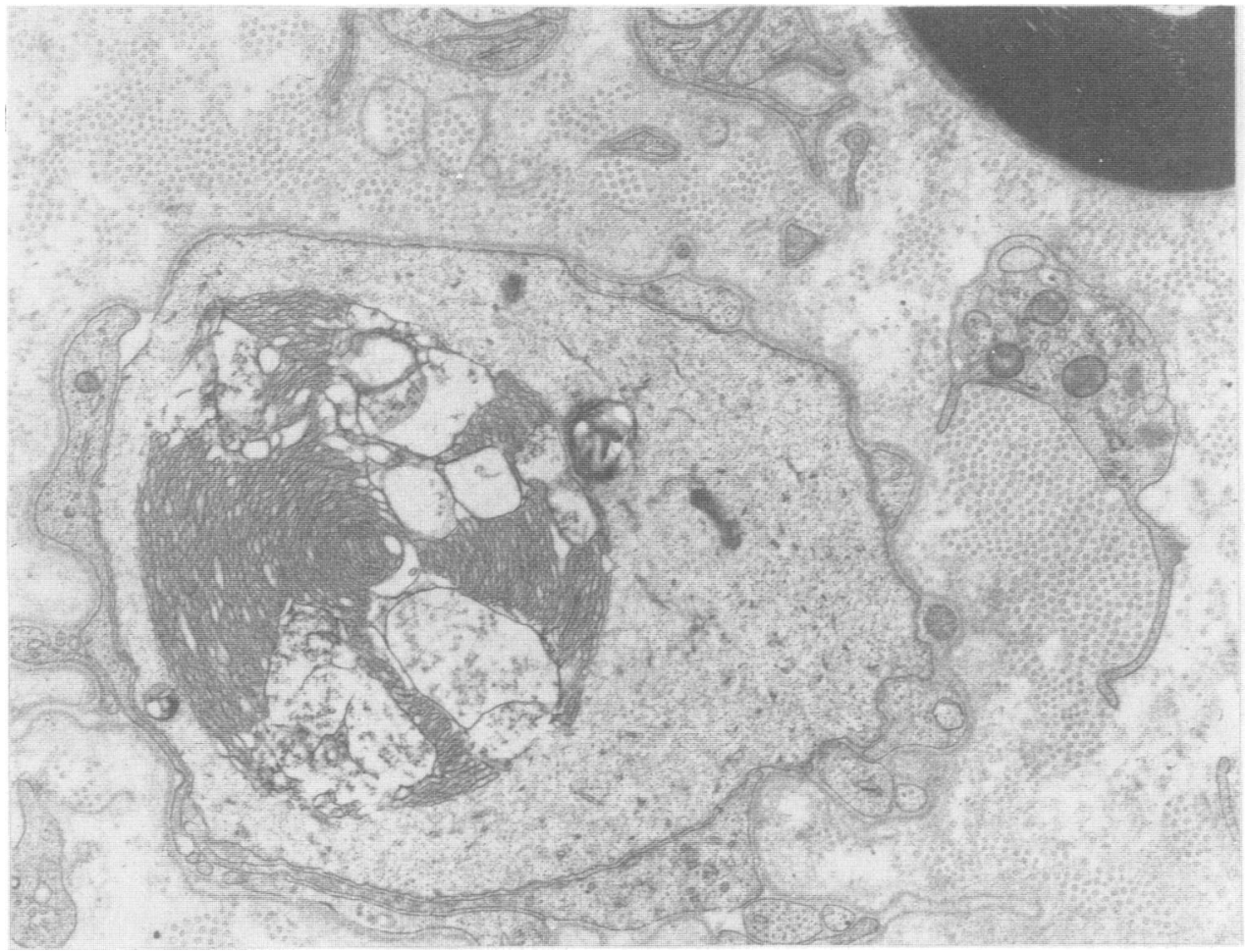

Fig 2 This fibre is severely damaged with unusual vesicular structures in the schwann cell cytoplasm. Several small axonal sprouts are visible at the periphery $(\times 15,000)$.

No changes of active demyelination and no inflammatory cells were seen.

Prednisone therapy $(60 \mathrm{mg} /$ daily $)$ led to great improvement, and the patient was able to walk with sticks after 3 weeks and was discharged on 21 October with a reduced dose of prednisone $(30 \mathrm{mg} /$ daily).

Ten days later a relapse occurred resulting in a motor deficit of all limbs, and the patient became quadriplegic in a few days. Prednisone was increased to $60 \mathrm{mg} /$ daily but without beneficial effect. Four 31 plasma exchanges given over $\mathbf{1 0}$ days gave a dramatic improvement. Motor strength was normal after the fourth pheresis. CSF protein at that time was $157 \mathrm{mg} / 100 \mathrm{ml}$. The patient was discharged. A third relapse occurred a month later with progressive flaccid quadriplegia and weakness of bulbar musculature. Four 31 plasma exchanges were given in 12 days. An improvement occurred again but less quickly than for the first cure. In the final days of December, the patient was able to walk unaided. On 9 January a fourth relapse occurred. Four 31 plasma exchanges given between 10 and 19 January were ineffective and in the following weeks the patient's condition continued to worsen. He was quadriplegic with dysphagia and respiratory failure requiring respiratory assistance. No improvement was noted over a period of 6 months. In July 1984 , he could be weaned off the respirator and a month later was fed normally. He remained quadriplegic with muscle wasting of the hands, but some movements of the right arm were noted. CSF protein was $170 \mathrm{mg} / 100 \mathrm{ml}$. The $\gamma$ globulins were high $(27 \%)$ without an oligoclonal pattern. His motor condition continued to improve but on 11 October 1984 he died suddenly. Necropsy was not permitted.

\section{Discussion}

These two patients had clinical and biological features of idiopathic chronic relapsing polyneuropathy. Repeated analysis, investigations and necropsy in case 1 did not disclose any paraproteinemia, porphyria, systemic disease or malignancy. The two patients died after a disease of 40 months duration for case 1 and 14 months for case 2.

Electrophysiological studies revealed abnormalities suggesting axonal lesions: fibrillation potentials at rest, reduction of amplitude of sensory potential, increase of nerve threshold excitability, and inexcitability of common peroneal nerve. The axonal pattern in case 2 was associated with a slowing of motor nerve conduction velocity, and in both cases with an increase of distal and proximal latencies.

A peripheral nerve biopsy performed in the two 


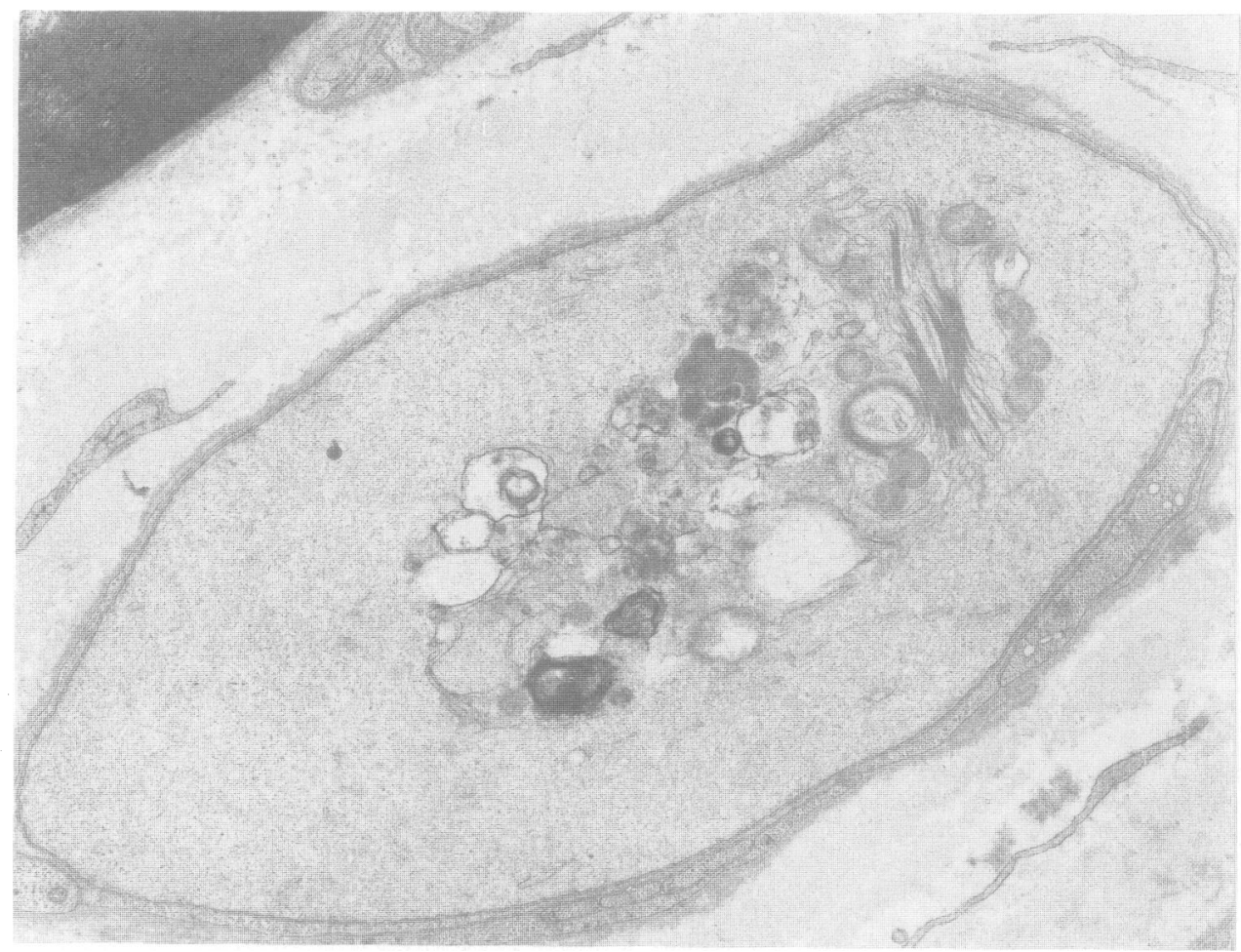

Fig 3 EM shows an enlarged axon containing abnormal mitochondria and vesicular structures $(\times 14,400)$. Two other small axons are present in the schwann cell cytoplasm $(\times 14,400)$.

patients showed an axonal loss of the largest myelinated fibres, clusters of regenerating axons; in case 2 very few enlarged axons were stripped of myelin. In neither case were any aspects of active demyelination observed as described in typical forms of GBS. ${ }^{5-8}$ The necropsy of case 1 did not disclose any inflammatory cells at the level of the nerve roots, unlike GBS.9 ${ }^{97}$ Axonal lesions have been observed in GBS but they appear to be secondary to the demyelinating process. ${ }^{10} 11$ Similar discoveries have been made in experimental allergic neuritis (EAN) by King and Thomas. ${ }^{12}$

Primary axonal lesions were observed by $\mathrm{Feasby}^{3}$ in a necropsied case of acute GBS. In this case the cervical and lumbar nerve roots exhibited severe axonal degeneration but, as in our case, there were no inflammatory cells or demyelination.

In GBS, demyelinating lesions are generally thought to be secondary to humoral factors. Saida et al ${ }^{13}$ have established that serum of animals with EAN contains a myelinotoxic factor. Cook and Dowling, ${ }^{14}$ and Saida et al ${ }^{15}$ have shown that the serum of patients with GBS has the same action. Using a complement fixation (C1) and transfer assay to measure antimyelin antibodies in
GBS sera, Koski et al $^{16}$ found antibody titres 6 to 56 times higher than in controls, and showed that the antineural antibody fibres fell precipitously after the early days of the illness. In this complement fixation assay, IgM appeared to be responsible for the high antineural antibody titres. Most antibody activity appeared to be directed against myelin components, but antibody was also detected against neurons and axons.

Although a few isolated remyelinating fibres were observed in our second case, axonal lesions in our observations were the main pathological feature, which suggests a different pathogenetic mechanism. The axonal lesions seen on the distal part of the biopsied nerve could be secondary to a demyelinating process affecting the roots, since electrophysiological studies indicated a proximal slowing of motor nerve conduction. However, in case 1 , the study of transverse spinal sections, which was performed at the cervical, thoracic and lumbar levels, did not disclose any demyelination. Furthermore, the cellular loss encountered in the posterior root ganglia and the anterior horns was slight and could not explain the axonal lesions observed on peripheral nerve biopsy. 
These findings seem to favour an initial axonal attack, possibly caused by a deleterious humoral factor. Plasma exchanges were given 6 and 2 months respectively after onset of the disease. In case 1 , improvement was slight and each relapse left the patient more and more disabled. In case 2 the first two relapses completely regressed in a few days, but then plasma exchanges were ineffective. Early relapses have been observed after plasma exchanges in acute polyneuropathies ${ }^{17}$ and in chronic relapsing forms. ${ }^{4}$

Outcome in polyneuropathy with axonal lesions is poor, and death occurred in our two patients. In the study of Feasby $e t \mathrm{al}^{3}$ one patient died, two remained severely handicapped and only one could work again. The three patients with axonal lesions reported by Pollard et $a l^{4}$ exhibited severe sensory and motor deficits with wasting. In chronic relapsing axonal polyneuropathy, it is generally believed that plasma exchanges are not of value. It is possible that factors involved in axonal lesions may be less influenced by exchanges than those causing demyelination, but other factors must be considered. American ${ }^{18}$ and French ${ }^{19}$ cooperative groups on plasma exchange in GBS have indicated that the delay between the onset of the disease and the start of pheresis has a bearing on outcome. Moreover, it is possible that axonal lesions, once constituted, continue to evolve independently of causative factors.

Chronic relapsing polyneuropathy with primary axonal lesions may have clinical and biological features similar to the demyelinating forms: motor deficit of all limbs, areflexia, albuminocytologic dissociation in the CSF, and respiratory failure, but muscle wasting is much more pronounced in the former and outcome is worse. Neuropathologic lesions are quite different since no active demyelinating lesions have been observed in any case. However, the second case exhibited a few features of isolated remyelinating fibres, which suggests a previous demyelinating process. Acute and chronic relapsing axonal polyradiculoneuropathy appear to be two clinical forms of a distinct entity different from GBS, despite some common clinical and biological features. The causative factors of such axonal lesions remain to be clarified.

\section{References}

1 Asbury AK. Criteria for diagnosis of Guillain-Barré syndrome. Ann Neurol 1978;3:565-7.
2 Prineas JW, McLeod JG. Chronic relapsing polyneuritis. $J$ Neurol Sci 1976;27:427-58.

3 Feasby TE, Gilbert JJ, Brown WF, et al. An acute axonal form of Guillain-Barré polyneuropathy. Brain 1986; 109:1115-26.

4 Pollard JD, McLeod JG, Gatenby P, Kronenberg H. Prediction of response to plasma exchange in chronic relapsing polyneuropathy. J Neurol Sci 1983;53: 269-87.

5 Arnason BGW. Polyradiculoneuropathies. In: Dyck PJ, Thomas PK, Lambert EH, Bunge R, eds. Peripheral Neuropathy. Philadelphia: Saunders, 1984;II: 2050-100.

6 Brechenmacher C, Vital C, Deminiere C, et al. GuillainBarré syndrome: An ultrastructural study of peripheral nerve in 65 patients. Clin Neuropathol 1987;6:19-24.

7 Prineas JW. Pathology of the Guillain-Barré syndrome. Ann Neurol 1981;9(suppl):6-19.

8 Vital C, Vallat JM. Ultrastructural Study of the Human Diseased Peripheral Nerve. New York: Masson, 1987;125-47.

9 Asbury AK, Arnason BGW, Adams RD. The inflammatory lesion in idiopathic polyneuritis. Its role in pathogenesis. Medicine (Baltimore) 1969;48:173-215.

10 Albers JW, Donofrio PD, McGonagle TK. Serial electrodiagnostic abnormalities in acute inflammatory demyelinating neuropathy. Muscle Nerve 1985;8: 528-39.

11 Dyck PJ, Karnes J, Lais A, et al. Pathologic alterations of the peripheral nervous system of humans. In: Dyck PJ, Thomas PK, Lambert EH, Bunge P, eds. Peripheral Neuropathy. Philadelphia: Saunders, 1984;I:760-870.

12 King RHM, Thomas PK, Pollard JD. Axonal and dorsal root ganglia cell changes in experimental allergic neuritis. Neuropathol Appl Neurobiol 1977;3:471-86.

13 Saida T, Saida K, Silberberg DH. Transfer of demyelination by intra neural injection of experimental allergic neuritis serum. Nature 1978;272:639-41.

14 Cook SD, Dowling PC. The role of auto antibody and immune complexes in the pathogenesis of the Guillain Barré syndrome. Ann Neurol 1981;9(suppl):70-9.

15 Saida T, Saida K, Lisak RP, et al. In vivo demyelinating activity of sera from patients with Guillain-Barré syndrome. Ann Neurol 1982;11:69-75.

16 Koski EL, Humphrey R, Shin ML. Anti peripheral myelin antibody in patients with demyelinating neuropathy: Quantitative and kinetic determination of serum antibody by $\mathrm{Cl}$ fixation. Proc Natl Acad Sci USA 1985;82:905-9.

17 Osterman PO, Fagius O, Safwenberg J, et al. Early relapses after plasma exchange in acute inflammatory polyradiculopathy. Lancet 1986;ii:1166.

18 The Guillain Barré Syndrome study Group-Plasmapheresis and acute Guillain Barré Syndrome. Neurology 1985;35:1096-104.

19 French Cooperative Group on plasma exchange in Guillain Barré syndrome. Role of replacement fluids. Ann Neurol 1987;22:753-61. 